\title{
Fault-tolerant analysis for active steering actuation system applied on conventional bogie vehicle
}

\author{
Bin Fu and Stefano Bruni \\ Dipartimento di Meccanica, Politecnico di Milano, Via La Masa 1, Milano, 20156, Italy \\ bin.fu@polimi.it
}

\begin{abstract}
Active steering system can drastically improve dynamic behaviors of the railway vehicle but will also introduce safety-critical issues. The fault-tolerant analysis therefore is essential for the design and implementation of this technology. In this work, an approach based on Risk Priority Number from Failure Mode and Effect Analysis is established to present quantitative assessment for fault tolerance of actuation system. This method is adopted to compare proposed nine different active steering schemes where two different hydraulic actuators are considered, and additional passive spring or redundant structure is implemented as back-up to ensure the safety. In case studies, the impacts of typical failure modes are investigated through multibody simulation and quantified by severity factor. Finally, the fault tolerance of different actuation schemes is compared by RPN values.
\end{abstract}

Keywords: active steering, fault tolerant, safety, FMEA, RPN

\section{Introduction}

Active suspension technology for railway vehicles has been studied since the $1970 \mathrm{~s}^{[1]}$ and continues drawing attention, with significant advances made over the following forty years ${ }^{[1,2,3]}$. Its beneficial effects have been validated from simulation to some field tests, but when it comes to the implementation, cost-benefit and safety-critical issues must be considered seriously.

The cost-benefit of active primary suspension is particularly attractive as this technology has the potential to provide substantial benefits in terms of lower maintenance costs and reduced impacts on wheels and the track. Active steering, as a main concept in active primary suspension, can drastically mitigate the wear between wheel and rail, increasing life cycle of vehicle and track system, and thus reducing maintenance cost. However, as active steering directly affects the kinematics of the wheelset, safety issues are concerned in case the actuation system fails in service. Therefore a fault-tolerant design of the active steering system is crucial for the implementation of the technology.

In this work, we propose an approach to analyzing the fault tolerance of active steering system where the concept of Risk Priority Number (RPN) from Failure Mode and Effect Analysis (FMEA) is adopted and multibody simulations are performed to study 
the impacts of failure modes. Based on this method, nine different active steering schemes are compared, and case studies are presented for further explanation.

\section{Methodology for fault-tolerant analysis}

\subsection{Failure Mode and Effect Analysis and Risk Priority Number}

Failure Mode and Effect Analysis (FMEA) is a systematic approach to evaluate the potential failure modes of the system and their effects ${ }^{[4]}$. It was firstly proposed for the design of aerospace craft and now has been successfully applied in many other industries to reduce the impacts of failure and to improve the reliability of the system. In FMEA, a core concept is calculating Risk Priority Number (RPN) which involves two essential factors: failure modes' severity and the likelihood, and also the third optional element: the ability to detect the failure modes. As is shown in Equation (1), the RPN is calculated as the multiplication of the levels of Severity, Occurrence and Detection which are characterized from 1 to 10 .

$$
R P N=\text { Severity } \times \text { Occurrence }(\times \text { Detection }) .
$$

In the background of active suspension system, if a frequent failure mode of actuation system has a severe impact on safety and is difficult to detect, the system needs to be improved in the design process to avoid this failure mode, for instance implementing redundant structures. A seminal work presented in Reference [5] adopted the RPN value to study the failures of active suspension for the first time. Following this work, we propose a quantitative method to assess the PRN value where the severity is focused upon as is introduced in Section 2.2.

\subsection{Vehicle dynamics simulation and severity level estimation}

The failure modes of actuation system have various impacts on vehicle and track systems which are hard to predict even with knowledge of vehicle dynamics. The simulation for vehicle and actuation system thus is necessary to investigate the failure effects.

In Europe, the vehicle acceptance norm EN 14363 presents the safety evaluation criterion by measuring two assessment quantities in the normal method: the sum of guiding forces of left and right wheels $\sum Y_{\max }$ and derailment coefficient $(Y / Q)_{\max }$. The detailed definitions, filtering methods and limits values of the two factors can be found in the standard ${ }^{[6]}$. When active suspension is failed, the increase of the assessment quantities and remaining margin from the limit value reflect the severity levels, and based on this, we establish the severity factor $s$ to quantify the impacts, as shown in Equation (2),

$$
\mathrm{s}(x)=\frac{x^{n}-F_{n}^{n}}{F_{l}^{n}-F_{n}^{n}}(n=2)
$$

where, $F_{n}$ is the value of safety factor $\left(\sum Y_{\max }\right.$ or $\left.(Y / Q)_{\max }\right)$ at normal condition; $F_{l}$ is the limit value of safety factor according to EN14363; $x$ is the factor's value to be measured in failure condition. An example of simulated derailment coefficient $x$ and corresponding severity factor $\mathrm{s}$ is shown in Figure 1 , where $F_{n}$ and $F_{l}$ are 0.3 and 0.8 respectively. When constant parameter $n$ is set to 2 , the gradient of severity $s$ over $x$ is 
increasing. In other words, the variation of severity factor will be more sensitive to $x$ when safety factors reach the value in a danger range. This weighted effect meets the common expectation for severity assessment. The factor $s$ can be used as an independent indicator for severity evaluation. When it is greater than 1 , the $\sum Y_{\max }$ or $(Y / Q)_{\max }$ exceeds the limit value, resulting in the chance of unsafety.

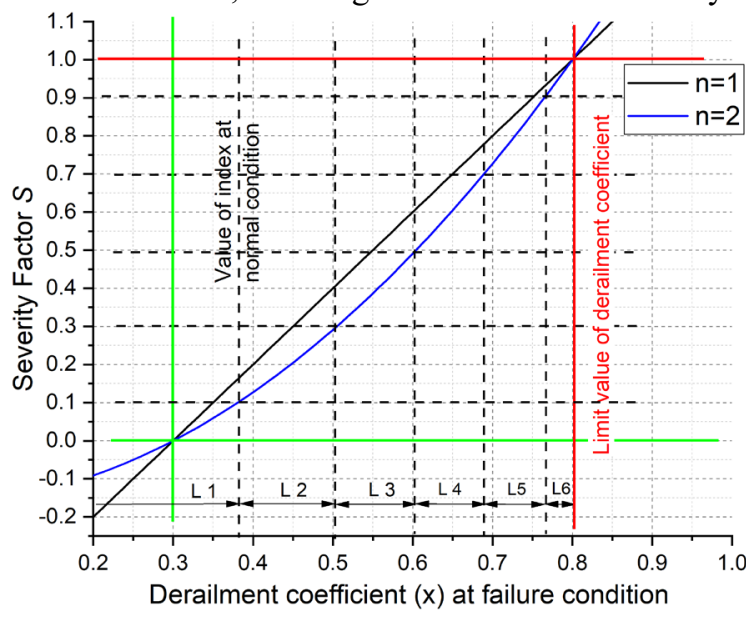

Fig. 1. Example of severity factor $s$ for derailment coefficient

In order to build the connection between the severity factor $s$ and Severity ranks for RPN calculation, 10 levels of severity are defined based on EN 60812 $2^{[4]}$, and the corresponding severity factor $s$ is graded as shown in Table 1. As the limit values of factors defined in EN 14363 are conservative for safety guarantee, the situation of " $\mathrm{s}=1$ " is not graded in the top level of severity but in Rank 7 that starts to have a risk of injured passengers and small chance of derailment.

Table 1. Description of severity levels for railway vehicle

\begin{tabular}{cccl}
\hline $\begin{array}{c}\text { Severity fac- } \\
\text { tor }\end{array}$ & $\begin{array}{c}\text { Severity } \\
\text { rank }\end{array}$ & Impact & \multicolumn{1}{c}{ Description } \\
\hline $\mathrm{s}<0.1$ & 1 & no impact & No recognizable effect \\
$0.1 \leq \mathrm{s}<0.3$ & 2 & very little & Noticed by few passengers \\
$0.3 \leq \mathrm{s}<0.5$ & 3 & little & Impacts on vehicle and infrastructure in long term \\
$0.5 \leq \mathrm{s}<0.7$ & 4 & very low & Noticed by many passengers \\
$0.7 \leq \mathrm{s}<0.9$ & 5 & low & Impacts on vehicle and infrastructure in mid term \\
$0.9 \leq \mathrm{s}<1.0$ & 6 & moderate & Impacts on vehicle and track in short term \\
\hline $\mathbf{1 . 0} \leq \mathrm{s}<1.1$ & 7 & high & Risk of injured people and small chance of derailment \\
$1.1 \leq \mathrm{s}<1.3$ & 8 & very high & Severe impacts on vehicle and infrastructure in short term \\
$1.3 \leq \mathrm{s}<1.5$ & 9 & very unsafe & Risk of many injured people and few dead people \\
$\mathrm{s} \geq 1.5$ & 10 & catastrophic & Risk of many dead people and the line closed for weeks \\
\hline
\end{tabular}


Then the Severity level can be obtained for calculating RPN. A method to quantify the Occurrence level is introduced in Section 4, whilst Detection is not considered in this work and estimating this factor has to do with the detailed implementation of the active steering system in a specific application.

\section{Simulation model}

\subsection{Brief introduction of Active steering schemes}

The principle of active steering is applying actuators between wheelsets and bogie in the longitudinal direction. Nine practical schemes labelled with "A1, A2, ., C3" are presented in three-by-three matrix as are shown in Fig. 2(a).

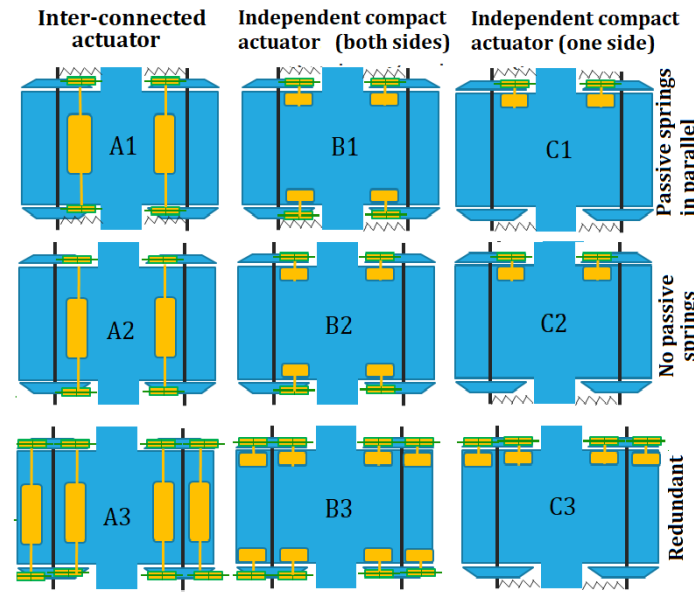

(a)

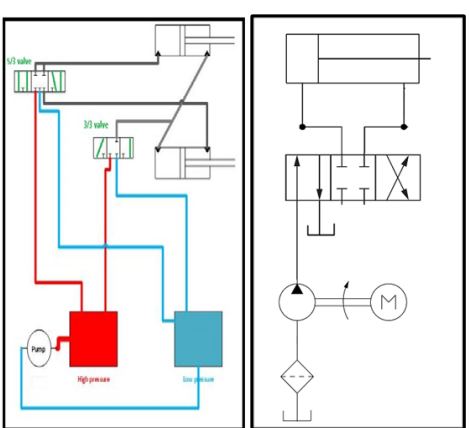

(b)

Fig. 2. The (a) "three by three" active steering schemes and (b)two types of actuator models

The schemes named with " $A$ " adopt inter-connected electro-hydraulic actuation system where the left and right actuators for each wheelset are combined in one system. By contrast, the schemes marked with " $\mathrm{B}$ " and " $\mathrm{C}$ " implement simple compact actuators for each wheel which is less likely to fail. The schematics of the inter-connected and compact hydraulic actuators are shown in Fig. 2(b): in both cases, the maximum force that can be generated by the actuator is $20 \mathrm{kN}$. For schemes "C", actuators are applied only on one side of the bogie whilst at the other side a passive suspension is used as a trade-off scheme between the benefits and the costs. These schemes therefore include a total number of actuators which is one half compared to the corresponding schemes " $\mathrm{B}$ ". The schemes in the first row with " 1 " include passive springs in parallel with actuators to enhance fault tolerance, but in these cases, higher actuator force is required to cancel out the action of passive springs. The schemes in the third row with " 3 " have redundant actuations as the back-up in case one fails in service so that the safety is expected to be ensured. The schemes " 2 " have neither passive springs nor redundant actuators. For each one of the 9 actuation schemes shown in Fig. 2 a dynamics model is built in Simulink Simscape. 
On curves, the control strategy for active steering is based on the ideal radial position of the wheelset in which the elongation of the actuator is controlled to force the wheelset in desired positions. On tangent track, the actuation system works in passive mode in which the compressibility and flow of hydraulic oil will generate stiffness and damping effects to mimic the behaviors of a passive primary suspension. The actuator response above $2 \mathrm{~Hz}$ is cut off to avoid introducing instability of the system.

\subsection{Brief introduction of vehicle model}

In this work, a new bogie with maximum operational speed $160 \mathrm{~km} / \mathrm{h}$ is designed as an integration of a conventional passive bogie and active steering system. The dynamics model of the passive vehicle is built in software SIMPACK and vehicle parameters are mainly originated from a real inter-city vehicle.

Two extreme simulation scenarios are configured to investigate the impacts of failure modes of actuation system. In Config. 1, the vehicle runs on tangent track at the maximum trial speed $176 \mathrm{~km} / \mathrm{h}(110 \%$ of max. operational speed), while in Config. 2 , the vehicle will negotiate tight curve $\mathrm{R} 250 \mathrm{~m}$ with super-elevation $150 \mathrm{~mm}$ at speed $73 \mathrm{~km} / \mathrm{h}$ (non-compensated lateral acceleration: $0.65 \mathrm{~m} / \mathrm{s}^{2}$ ). A measured track irregularity file is applied to both scenarios.

\section{Case studies for fault-tolerant analysis}

\subsection{Introduction of typical failure modes}

The active steering actuation system can be a complicated electro-hydraulic or electro-mechanical system. Each sub-structure, such as hydraulic actuator, valve, piping network, controller could have various failure mechanisms leading to the malfunction of actuation system. In spite of the complex failure causes, the failure modes could be summarized into limited items as is studied in Reference [5]. In this paper, we considered three typical failure modes: Maximum force, Zero force and Harmonic excitation. The Maximum force could come from the wrong signals of sensors and controllers where actuator will generate the maximum force to push or pull the wheelset. Zero force could be caused by a mechanical failure of the actuator or by severe leakage of hydraulic oil. In the vehicle dynamics model, a constant force $20 \mathrm{kN}$ and 0 are configured to simulate these two failure modes. The harmonic excitation could be the result of harmonic vibration from vehicle system, for instance the hunting motion of the wheelset. 


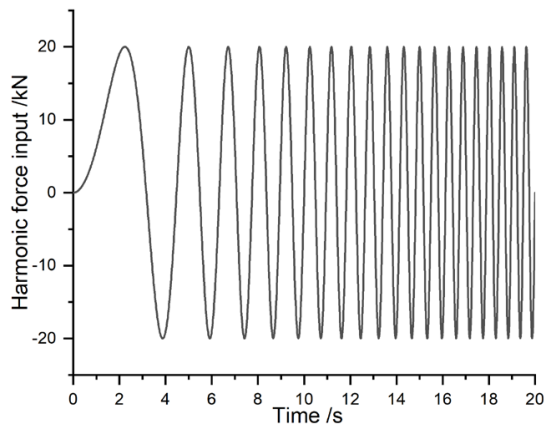

Fig. 3. Harmonic force input

As is shown in Fig. 3, a harmonic force input with varying frequency changing from $0 \mathrm{~Hz}$ to $2 \mathrm{~Hz}$ is applied in the model. In this work, the combination of different failure modes is not involved and we only consider one failure mode takes place on one actuation system.

\subsection{Case studies}

With the mentioned dynamics models and failure modes, the simulation was performed by means of co-simulation between SIMPACK and Simulink. Fig.4 presents the derailment coefficient and wheelset guiding force in Config. 1 at normal condition and with the failure Maximum force. The increase of factors from the normal condition to the failure condition is significant especially for schemes A2, B2 and C2 in which neither passive springs nor redundant actuators are implemented as the back-up. The values of factors then are processed to severity levels $s$ according to the method described in Section 2.2. The matrix $S$, as shown in Equation (3), is used to display the severity levels for the three-by-three actuation schemes. The first and second matrix in Equation (4) present the severity levels based on derailment coefficient and guiding force respectively and the higher severity level between the two is selected as the comprehensive assessment for Config.1, as shown in the third matrix.

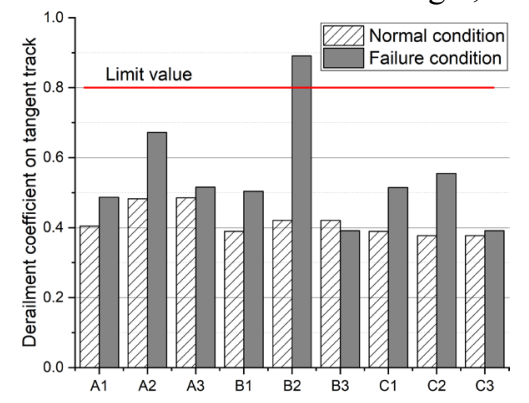

(a)

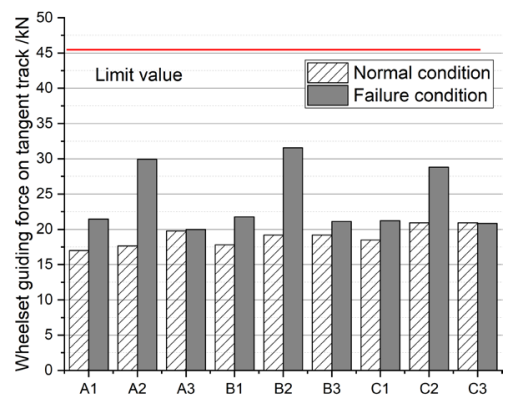

(b)

Fig. 4. (a)Derailment coefficient and (b) wheelset guiding force on tangent track (Config. 1)

$$
S=\left[\begin{array}{lll}
S_{A 1} & s_{B 1} & s_{C 1} \\
s_{A 2} & s_{B 2} & s_{C 2} \\
s_{A 3} & s_{B 3} & s_{C 3}
\end{array}\right]
$$




$$
S_{Y / Q, 1}=\left[\begin{array}{lll}
3 & 2 & 3 \\
4 & 8 & 3 \\
1 & 1 & 1
\end{array}\right] \quad S_{\sum Y, 1}=\left[\begin{array}{lll}
1 & 1 & 1 \\
3 & 3 & 2 \\
1 & 1 & 1
\end{array}\right] \quad S_{1}=\left[\begin{array}{lll}
3 & 2 & 3 \\
4 & 8 & 3 \\
1 & 1 & 1
\end{array}\right]
$$

With the same method, the simulation results in Config. 2 are presents in Fig. 5 and severity levels are calculated as shown in Equation (5), from which we conclude that the failure Maximum force has relatively limited impacts on safety when the vehicle negotiates the tight curve.

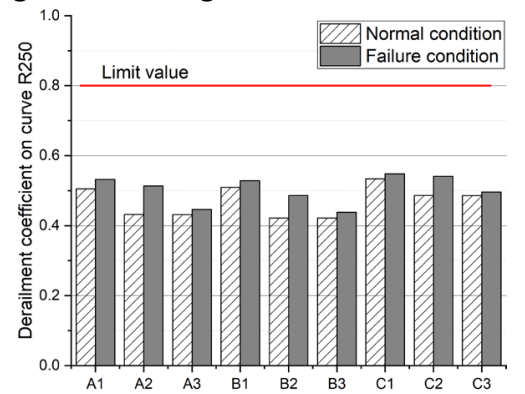

(a)

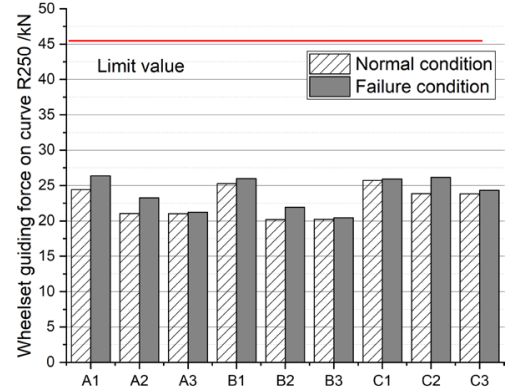

(b)

Fig. 5. (a)Derailment coefficient and (b) wheelset guiding force on curve R250 (Config. 2)

$$
S_{Y / Q, 2}=\left[\begin{array}{lll}
1 & 1 & 1 \\
2 & 2 & 2 \\
1 & 1 & 1
\end{array}\right] \quad S_{\sum Y, 2}=\left[\begin{array}{lll}
1 & 1 & 1 \\
1 & 1 & 1 \\
1 & 1 & 1
\end{array}\right] \quad S_{2}=\left[\begin{array}{lll}
1 & 1 & 1 \\
2 & 2 & 2 \\
1 & 1 & 1
\end{array}\right]
$$

Finally, for each one of the 9 configurations considered, the maximum value of the severity level obtained in the two running conditions is retained. The same procedure is repeated for the "Zero force" and "Harmonic excitation" failure modes and Table 2 presents the final results in terms of the severity levels obtained, whilst detailed results are not reported for the sake of brevity. The values in Table 2 show that the Max force is the most dangerous failure mode, followed by Harmonic excitation. Implementing redundant structure is effective to ensure the safety, while in absence of back-up for actuation system, the vehicle will be exposed in danger in some failure modes.

Table 2. Severity levels of different actuation schemes in three typical failure modes

\begin{tabular}{c|c|c|c|c|c|c}
\hline & \multicolumn{2}{|c|}{ Max force } & \multicolumn{2}{c|}{ Zero force } & \multicolumn{2}{c}{ Harmonic excitation } \\
\hline Schemes & Config 1 & Config 2 & Config 1 & Config 2 & Config 1 & Config 2 \\
\hline A1 & 3 & 1 & 1 & 1 & 2 & 1 \\
\hline B1 & 2 & 1 & 1 & 1 & 1 & 1 \\
\hline C1 & 3 & 1 & 1 & 1 & 2 & 1 \\
\hline A2 & 4 & 2 & 2 & 1 & 3 & 2 \\
\hline B2 & 8 & 2 & 2 & 1 & 5 & 3 \\
\hline C2 & 3 & 2 & 3 & 1 & 6 & 2 \\
\hline A3 & 1 & 1 & 1 & 1 & 1 & 1 \\
\hline B3 & 1 & 1 & 1 & 1 & 2 & 1 \\
\hline C3 & 1 & 1 & 1 & 1 & 1 & 1 \\
\hline
\end{tabular}


Regarding the estimation of failure modes' occurrence, we assume that the failure of each actuator is an independent event. For one single actuator, if its failure rate of a failure mode is $p_{a}$, then the failure rate of the whole actuation system for one bogie is calculated as $P=1-\left(1-p_{a}\right)^{n}$, where $n$ is the number of actuators implemented on one bogie. Based on EN 60812 and empirical data, we grade the levels of occurrence and failure rate as are shown in Table. 3.

Table 3. Ten levels of occurrence and failure rate (number of failure per operating hour)

\begin{tabular}{ccc}
\hline $\begin{array}{c}\text { Occurrence } \\
\text { Rank }\end{array}$ & Impact & Failure rate $[1 /$ hour $]$ \\
\hline 1 & & $\mathrm{P}<1 \times 10^{-8}$ \\
2 & Low & $1 \times 10^{-8} \leq \mathrm{P}<1 \times 10^{-7}$ \\
3 & & $1 \times 10^{-7} \leq \mathrm{P}<5 \times 10^{-7}$ \\
\hline 4 & & $5 \times 10^{-7} \leq \mathrm{P}<2 \times 10^{-6}$ \\
5 & Moderate & $2 \times 10^{-6} \leq \mathrm{P}<8 \times 10^{-6}$ \\
6 & & $8 \times 10^{-6} \leq \mathrm{P}<3 \times 10^{-5}$ \\
\hline 7 & High & $3 \times 10^{-5} \leq \mathrm{P}<1 \times 10^{-4}$ \\
8 & & $1 \times 10^{-4} \leq \mathrm{P}<3 \times 10^{-4}$ \\
\hline 9 & Very high & $3 \times 10^{-4} \leq \mathrm{P}<1 \times 10^{-3}$ \\
10 & & $\mathrm{P} \geq 3 \times 10^{-3}$ \\
\hline
\end{tabular}

Table 4 gives the estimated probabilities of the failure modes which are then used to define the RPN values. As the failure Zero force is much related to mechanical structure of actuators, we speculate the complicated inter-connected actuator is much more likely to cause this failure then the simple compact actuator. With these values and described method we obtained the levels of Occurrence for the failure modes on one bogie, as are shown in Equation (6). When redundant actuators are applied, the increasing number of actuators causes the higher failure rate for each bogie, but this increased failure rate at most leads to one-step rise of Occurrence level.

Table 4. probability of failure modes on each actuator [1/hour]

\begin{tabular}{ccc}
\hline Failure modes & Inter-connected actuator & Independent compact actuator \\
\hline Max force & $5 \times 10^{-7}$ & $2 \times 10^{-7}$ \\
Zero force & $1 \times 10^{-6}$ & $1 \times 10^{-7}$ \\
Harmonic excitation & $2 \times 10^{-7}$ & $2 \times 10^{-7}$ \\
\hline$O_{\text {max }_{-} f}=\left[\begin{array}{lll}4 & 4 & 3 \\
4 & 4 & 3 \\
4 & 4 & 4\end{array}\right] \quad O_{\text {zero } f}=\left[\begin{array}{lll}4 & 3 & 3 \\
4 & 3 & 3 \\
5 & 4 & 3\end{array}\right] \quad O_{\text {harmoic }}=\left[\begin{array}{lll}3 & 4 & 3 \\
3 & 4 & 3 \\
4 & 4 & 4\end{array}\right]$
\end{tabular}

The estimation for Detection level is not involved in this work, therefore the Severity level and Occurrence level are multiplied for RPN calculation, see the results in Table 5. This table clearly reveals the impacts of failure modes and also the capability of fault tolerance of different actuation schemes. Apart from making comparisons between 
different schemes, the RPN value can also be used to evaluate the improvement between the previous and modified schemes in the design process.

It should be noted that the severity of failure modes is not only determined by the active actuation system, but also affected by the passive suspension. The failure mode Zero force in above simulation shows limited degradation of dynamic behaviors, because in the original passive bogie, the anti-hunting damper and the coil spring above the axle-box still ensure a good stability when most part of the primary longitudinal stiffness is lost at one side of wheelset, while if the anti-hunting damper is removed, the failure Zero force will have a much more severe impact at high speed.

Table 5. RPN values of different actuation schemes in three typical failure modes

\begin{tabular}{c|c|c|c|c|c|c}
\hline & \multicolumn{2}{|c|}{ Max force } & \multicolumn{2}{c|}{ Zero force } & \multicolumn{2}{c}{ Harmonic excitation } \\
\hline Schemes & Config 1 & Config 2 & Config 1 & Config 2 & Config 1 & Config 2 \\
\hline A1 & 12 & 4 & 4 & 4 & 6 & 3 \\
\hline B1 & 8 & 4 & 3 & 3 & 4 & 4 \\
\hline C1 & 9 & 3 & 3 & 3 & 6 & 3 \\
\hline A2 & 16 & 8 & 8 & 4 & 9 & 6 \\
\hline B2 & 32 & 8 & 6 & 3 & 20 & 12 \\
\hline C2 & 9 & 6 & 9 & 3 & 18 & 6 \\
\hline A3 & 4 & 4 & 5 & 5 & 4 & 4 \\
\hline B3 & 4 & 4 & 4 & 4 & 8 & 4 \\
\hline C3 & 4 & 4 & 3 & 3 & 4 & 4 \\
\hline
\end{tabular}

\section{Conclusion}

In this work, we proposed a method to evaluate the severity of different failure modes and fault-tolerant capability of different actuation schemes. The quantified severity factor $s$ and RPN value can provide a good way for assessing different active steering schemes. Implementing a redundant actuation scheme is an effective method to improve the fault tolerance of actuation system, whilst in absence of any redundancy or passive back-up the vehicle may be exposed to unsafe running conditions in case of particularly dangerous failure modes taking place. Future extension of this work will be addressed to the validation of the procedure established to define severity and occurrence levels and to extending the analysis to other failure modes.

\section{Acknowledgment}

The work presented in this paper has received funding from the Shift2Rail Joint Undertaking under the European Union's Horizon 2020 research and innovation programme under grant agreement no. 777564. The contents of this publication only reflect the authors' view and the Joint Undertaking is not responsible for any use that may be made of the information contained in the paper. 


\section{References}

1. R. M. Goodall and W. Kortüm, "Active Controls in Ground Transportation-Review of the State-of-the-Art and Future Potential," Veh. Syst. Dyn., vol. 12, no. 4-5, pp. 225-257, 1983.

2. R. Goodall, "Active railway suspensions: Implementation status and technological trends," Veh. Syst. Dyn., vol. 28, no. 2-3, pp. 87-117, 1997.

3. S. Bruni, R. Goodall, T. X. Mei, and H. Tsunashima, "Control and monitoring for railway vehicle dynamics," Veh. Syst. Dyn., vol. 45, no. 7-8, pp. 743-779, 2007.

4. BS EN IEC 60812:2018 BSI: Failure modes and effects analysis (FMEA and FMECA), British Standards Institution, 2018.

5. A. Qazizadeh, S. Stichel, and R. Persson, "Proposal for systematic studies of active suspension failures in rail vehicles," Proc. Inst. Mech. Eng. Part F J. Rail Rapid Transit, vol. 232, no. 1, pp. 199-213, 2018.

6. BS EN14363:2016. Railway applications testing for the acceptance of running characteristics of railway vehicles testing of running behaviour and stationary tests, British Standards Institution, 2016. 